\title{
Evaluation of approximate Fuzzy Membership Function using linguistic input-an approached based on cubic spline
}

\author{
Stabania Chowdhury ${ }^{\mathrm{a}} \&$ Rahul Kar ${ }^{\mathrm{b}, *}$ \\ ${ }^{a}$ Department of Mathematics, Guru Nanak Institute of Technology, Kolkata, India \\ ${ }^{b}$ Department of Mathematics, Springdale High School, Kalyani, India
}

\begin{abstract}
Fuzzy logic systems have found extensive use in system identification, decision making, and pattern recognition problems from industries to academics. The membership functions play a pivotal role in overall role in fuzzy representation, as these are considered as the building blocks of fuzzy set theory and they decide the degree of truth in fuzzy logic. The extraction of the membership function is ambience dependent and thus complication exists in the process of evaluation. In this assessment the main work deals with the derivation of fuzzy membership function where numerical data is available. The numerical cubic spline and defuzzification technique are used here. In this paper we mainly used triangular fuzzy number to construct the membership function. A case study is furnished to emphasize the advantage of adopting the method.
\end{abstract}

(C) 2020 Author(s). All rights reserved.

Keywords: Fuzzy membership function; Defuzzification; Cubic spline formula; Risk factor.

\section{Introduction}

Fuzzy logic systems have found extensive use in system identification, decision making, and pattern recognition problems from industries to academics. The membership functions play a pivotal role in overall role in fuzzy representation, as these are considered as the building blocks of fuzzy set theory and they decide the degree of truth in fuzzy logic. The membership function was introduced by Lofti A. Zadeh (Zadeh, 1988). As there are infinite numbers of ways to characterize fuzziness, so there are infinite number of ways to evaluate membership functions. Thus, evaluation of membership function hugely depends on problem size and context of the problem. Relying on personal intuition and experience of the researchers/individual it becomes quite challenging to exclude the inherent uncertainties in this process. Membership functions are used to represent fuzzy set graphically. Universe of discourse is represented by $\mathrm{x}$ axis, whereas the $\mathrm{y}$ axis represents the degree of membership in the $[0,1]$ interval. The base set is the deciding factor for the type of representation of the membership function. Because of the simplicity and efficiency with respect to the computability, generally piecewise linear membership functions are preferred. The evaluation of membership function in fuzzy logic involves following steps. At first, the crisp set is transformed to fuzzy set or fuzzy set to fuzzier set. The process is called fuzzification and it deals with translating crisp input to linguistic variables. Support fuzzification and grade fuzzification are different types of fuzzification. Now, the next step is to convert a fuzzy set/member into a crisp set/ member. This rounding off is called defuzzification. Different methods of defuzzification that are employed are Max-membership, centroid method, weighted average method, mean -max method etc. An interpolation method is carried out subsequently. Often preference is given to spline interpolation method over polynomial interpolation owing to considerate decrease in interpolation error even to low degree

\footnotetext{
* Corresponding author.

E-mail address: rkar997@gmail.com (Rahul Kar)
} 
polynomials, in spline interpolation method, the interpolate is a special type of piece wise polynomial called spline, classically, cubic splines having degree 3 are used which are able to achieve the continuity of first derivative but not of second derivative. In these present times bike speed and risk has increased exponentially in few years. So, considering the severity of the issue, the present works deals in finding the membership function of the set 'Risk' of a certain speed while riding on a bike in a standard road.

\section{Preliminaries}

A fuzzy set is a pair $(\mathrm{U}, \mathrm{m})$ where $\mathrm{U}$ is a set and $m=\mu_{A}$ : $\mathrm{U}-[0.1]$ a membership function . The reference set $\mathrm{U}$ (sometimes denoted by $\mathrm{X}$ ) is called universe of discourse, and for each $\mathrm{X}$ belongs $\mathrm{U}$, the value $\mathrm{m}(\mathrm{x})$ is called the grade of membership of $\mathrm{x}$ in $(\mathrm{U}, \mathrm{m})$. The function $\mathrm{m}=\mu_{A}$ is called the membership function of the fuzzy set $\mathrm{A}=(\mathrm{U}, \mathrm{m})$.

\section{Defuzzification}

This process, which involves the translation of fuzzy quantity into crisp quantities called defuzzification. Different methods are proposed in the literature of Van Leekwijck \& Kerre (1999). Most of the methods are basically extended form of centre of gravity method or fuzzy mean method. The choice of defuzzification method is context or problem dependant. According to Hellendoorn and Thomas (12), there are five categories against which methods are measured. These are: Continuity., Disambiguates, Plausibility, Computational simplicity.Weighing method.

If $\mathrm{A}=\left(a_{1}, a_{2}, a_{3}\right)$ is triangular fuzzy number, them by signed distance method, defuzzified value of $\mathrm{A}$ is given by

$$
\begin{gathered}
\mathrm{d}(\mathrm{A})=\frac{1}{2} \int_{0}^{1}\left[A_{L}\left((\alpha)+A_{R}(\alpha)\right] \mathrm{d} \alpha, \text { where } \alpha \varepsilon[0,1]\right. \\
A_{L}(\alpha)=a_{1}+\left(a_{2}-a_{1}\right) \alpha, A_{R}(\alpha)=a_{3}+\left(a_{3}-a_{2}\right\} \\
\mathrm{d}(\mathrm{A})=\frac{1}{4}\left(a_{1}+2 a_{2}+a_{3}\right)
\end{gathered}
$$

\section{Cubic Spline Interpolation}

Spline interpolation technique has been developed in recent times and has found very useful application in numerical differentiation, integration and solution of boundary value problems also. The term spline has been used because of its similarity in the technique used by a draftsman. A draftsman draws a smooth curve through a given set of points called knots in such a way that not only the curve is continuous but it's slope and curvature are also continuous; A spline interpolation attempts to interpolate a function between a given set of points by means of piecewise smooth polynomials (Panda \& Dash, 2006).

\section{Literature Review}

Lambert (1992) in his work, had investigated the effectiveness of applying the analytic hierarchy decision model of TL sooty for specification of membership values of fuzzy set. Norwich \& Turksen (1984), had addressed the problem of measurement of fuzziness. They also suggested a measurement model in order-dense domain of discourse. Choi \& Rhee (2009) had proposed three novel interval type-2 fuzzy membership function (IT2 FMF) generation methods. The methods were based on heuristics, histograms, and interval type-2 fuzzy $C$-means. The performance of the methods is evaluated by applying them to back-propagation neural networks (BPNNs). Zhu et. al. (2010) used in their paper that Fuzzy membership function is an effective tool to represent relationship between soil and environment for predictive soil mapping. Usually construction of a fuzzy membership function requires knowledge on soil-landscape relationships obtained from local soil experts or from extensive field samples. Monicka, Sekhar, \& Kumar (2011) tried to evaluate the performance of Membership Functions on Fuzzy Logic Controlled AC Voltage Controller for Speed Control of Induction Motor Drive. The membership functions selected for evaluation are triangular, trapezoidal, Gaussian and bell-shaped functions. The different membership function evaluation is done considering seven linguistic sets for error and change in error. Mandal, Choudhury, \& Chaudhuri (2012) made a search of suitable fuzzy membership function in prediction of time series data. In this paper they make an effort to predict the time 
series data based on different fuzzy membership functions like Gaussian, Triangular, S-function, trapezoidal, Gbell, Dsigmoidal, Psigmoidal and Pie-shaped. They have been made a comparison among the predicted data using different membership functions. One membership function has been selected based on minimum error in prediction of data. Lam \& Lauber (2013) investigated the stability of fuzzy-model-based (FMB) control system, formed by a T-S fuzzy model and a fuzzy controller connected in a close loop, based on a fuzzy-Lyapunov function. A general FMB control system that the T-S fuzzy model and fuzzy controller not sharing the same premise membership functions and/or the same number of fuzzy rules is considered. Piegat \& Landowski. (2015) proposed a horizontal membership function with its applications. They define a fuzzy set not in form of commonly used vertical membership functions of type $\mu=f_{1}(x)$ but in the horizontal form $x=f_{2}(\mu)$. Omrane, Masmoudi, \& Masmoudi (2016) described in their paper the design and the implementation of a trajectory tracking controller using fuzzy logic for mobile robot to navigate in indoor environments. The main contribution of the paper can be summarized in the fact that we use only one fuzzy controller for navigation and obstacle avoidance. Harliana \& Rahim (2017) they made a comparative analysis of membership function on Mamdani fuzzy inference system for decision making. Lagunes, Castillo, \& Soria (2018) proposed a work optimization of parameters of the membership functions in fuzzy control systems allows a better performance of the actuators that are controlling an autonomous robot. That article had explained the proposed methodology for the optimization of parameters of membership functions of a tracking fuzzy controller for a mobile autonomous robot using the firefly algorithm. Kar \& Mukherjee (2019) proposed a new method of fuzzy membership function evaluation by interpolation and defuzzification. Their work deals with the extraction of fuzzy membership function where statistical data is available. The methodology is based on numerical interpolation and defuzzification.

\section{Methodology}

Let us consider a fuzzy set $A$ where membership function $\mathrm{u}_{\mathrm{c}} A$ (x) is to be determined. The methodology is stepwise demonstrated as follows:

Step1:

A set of $\mathrm{n}$ points $x_{i}(i=1,2,3, \ldots \ldots \ldots, n ; n \in \square)$ is selected from the set $A$.The points may or may not be equispaced.

Step2:

A point scale of finite number of Linguistic Terms (LTs) is constructed. We denote the scale as $L=\left\{(L T)_{l} ; l=1,2,3, \ldots . ., n\right\}$.To each $\mathrm{LT}$ in the scale, there is associated a Triangular Fuzzy Numbers (TFN).Let $T_{i}$ denote the TFN corresponding to the linguistic term $(L T)_{l} ; l=1,2,3, \ldots . ., n$ where $T_{i}=\left(a_{i}, b_{i}, c_{i}\right)$

Step3:

Now a survey is to be conducted among a satisfactory population to collect data for the evaluation of membership function of $A$.Let the survey consist of $\mathrm{N}$ number of data. To be more realistic; the data are collected in terms of Linguistic terms from the set $L$.So at the completion of the survey, we must have N TFN's for each point $x_{i}$.

\section{Step4:}

Next the task is to be assembled the all the TFN's for a particular point. Let $\left\{\stackrel{\square}{ }_{i}^{k}=\left(a_{i}^{k}, b_{i}^{k}, c_{i}^{k}\right) ; k=1,2,3, \ldots . ., N\right\}$ is the set of $\mathrm{N}$ TFNs which are to be assembled into one. This will be executed by the Min-Avg-Max principle. 


$$
\begin{array}{lr}
a_{i}=\underset{k}{\operatorname{Min}}\left\{a_{i}^{k}\right\} \\
\text { So we extract only one TFN } \stackrel{\square}{T}_{i}=\left(a_{i}, b_{i}, c_{i}\right) \text { for the point } x_{i}, i=1,2,3, . ., n \text { where } & b_{i}=\frac{1}{N} \sum_{k=1}^{N} b_{i}^{k} \\
c_{i}=\underset{k}{\operatorname{Max}}\left\{c_{i}^{k}\right\}
\end{array}
$$

\section{Step6:}

Now convert each TFN i.e. $\stackrel{\square}{T}_{i}=\left(a_{i}, b_{i}, c_{i}\right)$ to its corresponding risk $y_{i} ; i=1,2,3, \ldots \ldots, n$

\section{Application}

Now a survey is to be conducted among a satisfactory population to collect data for the evaluation of the membership function of a fuzzy set 'Risk' of a certain speed while riding on a bike in a standard road. The aim is to find the membership polynomial using Lagrange's polynomial formula. A group of 50 bike riders from different geographical locations is selected and they are asked to submit their opinion on the risk factor of bike riding at specified speeds in standard road conditions. To avoid crisp errors, the data are collected in terms of Linguistic Terms.

\section{Step1:}

10 equispaced speeds $(\mathrm{km} / \mathrm{h})$ of the bike are taken as follows 10, 20, 30, 40, 50, 60, 70, 80, 90, 100.

\section{Step2:}

A point scale of finite number of Linguistic Terms (LTs) is constructed as: Very Low (VL) , Low (L), Medium low (ML), Medium (M), Medium High (MH), High (H), Very High (VH). To each LT in the scale, there is associated a Triangular Fuzzy Numbers (TFN).

Table 1. Collected data from 50 persons

\begin{tabular}{ccc}
\hline $\begin{array}{c}\text { Speed } \\
\mathrm{Km} / \mathrm{h}\end{array}$ & Measurement (no. Of person who say this) & TFN \\
\hline $0-10$ & VL (50) & $(1,1,2)$ \\
$10-20$ & VL (50) & $(1,1,2)$ \\
$20-30$ & VL (48), L (2) & $(1,1.08,4)$ \\
$30-40$ & L (40), ML (10) & $(2,3.2,5)$ \\
$40-50$ & L (17), ML (23), M (10) & $(2,3.86,6)$ \\
$50-60$ & ML (7), M (37), MH (6) & $(3,5.1,8)$ \\
$60-70$ & MH (33), H (16), VH (1) & $(6,7.38,10)$ \\
$70-80$ & H (10), VH (40) & $(7,9.6,10)$ \\
$80-90$ & VH (50) & $(9,10,10)$ \\
$90-100$ & VH (50) & $(9,10,10)$ \\
\hline
\end{tabular}

\section{Step3:}

Next the task is to assemble all the TFN's for a particular point. Let $\left\{T_{i}^{{ }^{k}}=\left(a_{i}^{k}, b_{i}^{k}, c_{i}^{k}\right) ; k=1,2,3, \ldots ., N\right\}$ is the set of N TFNs which are to be assembled into one. This will be executed by the Min-Avg-Max principle. 
Step4:

$$
\begin{array}{lr}
a_{i}=\underset{k}{\operatorname{Min}}\left\{a_{i}^{k}\right\} \\
\text { So we extract only one TFN } \stackrel{\square}{T}_{i}=\left(a_{i}, b_{i}, c_{i}\right) \text { for the point } x_{i}, i=1,2,3, . ., n \text { where } & b_{i}=\frac{1}{N} \sum_{k=1}^{N} b_{i}^{k} \\
c_{i}=\underset{k}{\operatorname{Max}}\left\{c_{i}^{k}\right\}
\end{array}
$$

Now convert each TFN i.e. $\stackrel{\square}{T}_{i}=\left(a_{i}, b_{i}, c_{i}\right)$ to its corresponding risk $y_{i} ; i=1,2,3, \ldots \ldots, n$

Table 2. Defuzzified values and their Normalised scores

\begin{tabular}{cccc}
\hline $\begin{array}{c}\text { Speed } \\
\mathrm{Km} / \mathrm{h}\end{array}$ & Mid point & Risk & $\begin{array}{c}\text { Normalized } \\
\text { values }\end{array}$ \\
\hline $0-10$ & 5 & 1.25 & 0.128 \\
$10-20$ & 15 & 1.25 & 0.128 \\
$20-30$ & 25 & 1.79 & 0.184 \\
$30-40$ & 35 & 3.35 & 0.344 \\
$40-50$ & 45 & 3.93 & 0.403 \\
$50-60$ & 55 & 5.3 & 0.544 \\
$60-70$ & 65 & 7.69 & 0.789 \\
$70-80$ & 75 & 9.05 & 0.928 \\
$80-90$ & 85 & 9.75 & 1.000 \\
$90-100$ & 95 & 9.75 & 1.000 \\
\hline
\end{tabular}

Step 5

$$
f(x)= \begin{cases}5.3506 \cdot 10^{-6} \cdot x^{3}-8.0259 \cdot 10^{-5} \cdot x^{2}-1.3376 \cdot 10^{-4} \cdot x+1.3001 \cdot 10^{-1}, & \text { if } x \in[5,15], \\ 2.9247 \cdot 10^{-5} \cdot x^{3}-1.1556 \cdot 10^{-3} \cdot x^{2}+1.5996 \cdot 10^{-2} \cdot x+4.9356 \cdot 10^{-2}, & \text { if } x \in(15,25], \\ -7.4339 \cdot 10^{-5} \cdot x^{3}+6.6133 \cdot 10^{-3} \cdot x^{2}-1.7823 \cdot 10^{-1} \cdot x+1.6679, & \text { if } x \in(25,35], \\ 6.3108 \cdot 10^{-5} \cdot x^{3}-7.8185 \cdot 10^{-3} \cdot x^{2}+3.2689 \cdot 10^{-1} \cdot x-4.2251, & \text { if } x \in(35,45], \\ 4.9085 \cdot 10^{-6} \cdot x^{3}+3.8348 \cdot 10^{-5} \cdot x^{2}-2.6671 \cdot 10^{-2} \cdot x+1.0783, & \text { if } x \in(45,55], \\ -6.0742 \cdot 10^{-5} \cdot x^{3}+1.0871 \cdot 10^{-2} \cdot x^{2}-6.2245 \cdot 10^{-1} \cdot x+1.2001 \cdot 10^{1}, & \text { if } x \in(55,65], \\ 2.8058 \cdot 10^{-5} \cdot x^{3}-6.4452 \cdot 10^{-3} \cdot x^{2}+5.0308 \cdot 10^{-1} \cdot x-1.2386 \cdot 10^{1}, & \text { if } x \in(65,75], \\ -1.2489 \cdot 10^{-5} \cdot x^{3}+2.6777 \cdot 10^{-3} \cdot x^{2}-1.8114 \cdot 10^{-1} \cdot x+4.7199, & \text { if } x \in(75,85], \\ 1.6898 \cdot 10^{-5} \cdot x^{3}-4.8159 \cdot 10^{-3} \cdot x^{2}+4.5582 \cdot 10^{-1} \cdot x-1.3327 \cdot 10^{1}, & \text { if } x \in(85,95] .\end{cases}
$$

\section{Conclusion}

As the extraction of the membership function is ambience dependent and thus no exact methods for the derivation are not exists. This method facilitates the problem where numerical data are available. The above procedure and discussion can be continuing for further investigation where a smaller number of data is available and also applied to other momentous problems of membership function evaluation. 


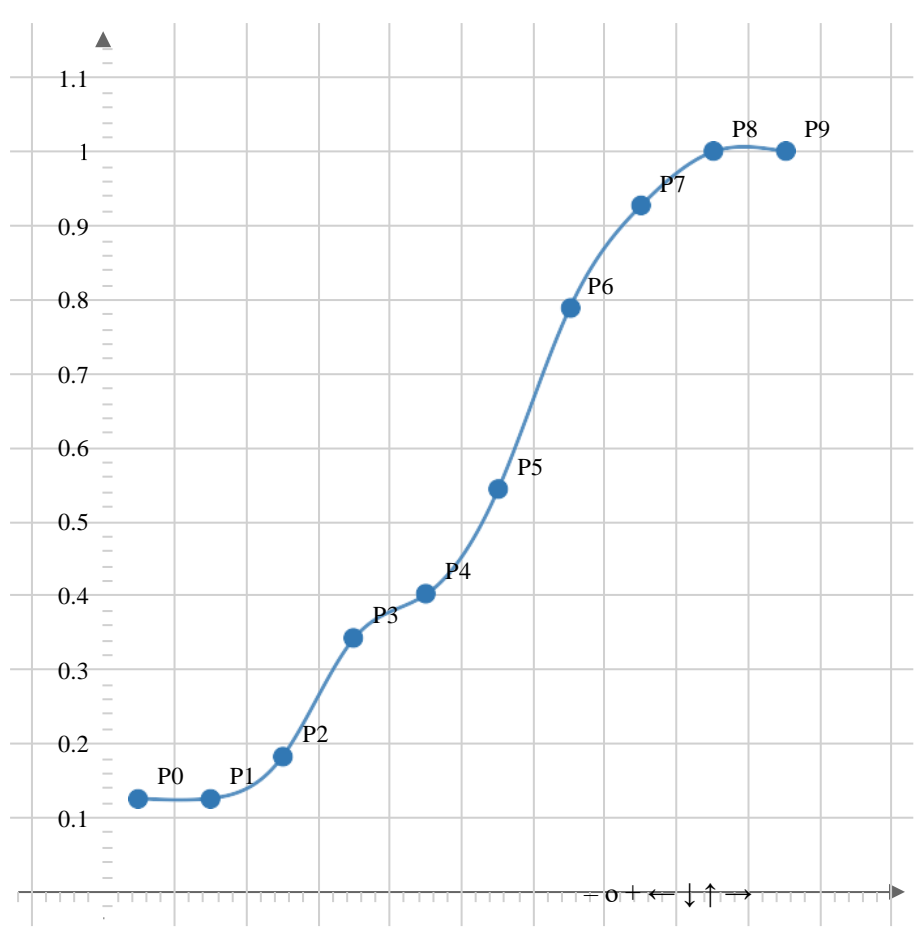

Fig. 1. Risk-Speed Graph

\section{References}

Choi, B. I., \& Rhee, F. C. H. (2009). Interval type-2 fuzzy membership function generation methods for pattern recognition. Information Sciences, 179(13), 2102-2122.

Harliana, P. \& Rahim, R. (2017). Comparative analysis of membership function on Mamdani fuzzy inference system for decision making. In Journal of Physics Conference Series (Vol. 930, No. 1, p. 012029).

Kar, R \& Mukherjee, S (2019). A New Method of Fuzzy Membership Function Evaluation by Interpolation and Defuzzification. International Journal of Innovative Knowledge Concepts, 7(S1).

Lagunes, M. L., Castillo, O., \& Soria, J. (2018). Optimization of membership function parameters for fuzzy controllers of an autonomous mobile robot using the firefly algorithm. In Fuzzy Logic Augmentation of Neural and Optimization Algorithms: Theoretical Aspects and Real Applications (pp. 199-206). Springer, Cham.

Lam, H. K., \& Lauber, J. (2013). Membership-function-dependent stability analysis of fuzzy-model-based control systems using fuzzy Lyapunov functions. Information Sciences, 232, 253-266.

Lambert, J. M. (1992). The fuzzy set membership problem using the hierarchy decision method. Fuzzy sets and systems, 48(3), 323-330.

Mandal, S. N., Choudhury, J. P., \& Chaudhuri, S. B. (2012). In search of suitable fuzzy membership function in prediction of time series data. International Journal of Computer Science Issues, 9(3), 293-302.

Monicka, J. G., Sekhar, N. G., \& Kumar, K. R. (2011). Performance evaluation of membership functions on fuzzy logic controlled ac voltage controller for speed control of induction motor drive. International Journal of Computer Applications, 13(5), 8-12. 
Norwich, A., \& Turksen, I. B. (1984). A model for the measurement of membership and the consequences of its empirical implementation. Fuzzy sets and systems, 12(1), 1-25.

Omrane, H., Masmoudi, M. S., \& Masmoudi, M. (2016). Fuzzy logic based control for autonomous mobile robot navigation. Computational intelligence and neuroscience, 2016.

Panda, R., \& Dash, M. (2006). Fractional generalized splines and signal processing. Signal Processing, 86(9), 23402350.

Piegat, A., \& Landowski, M. (2015). Horizontal membership function and examples of its applications. International Journal of Fuzzy Systems, 17(1), 22-30.

Van Leekwijck, W., \& Kerre, E. E. (1999). Defuzzification: criteria and classification. Fuzzy sets and systems, 108(2), 159-178.

Zadeh, L. A. (1988). Fuzzy logic. Computer, 21(4), 83-93.

Zhu, A. X., Yang, L., Li, B., Qin, C., Pei, T., \& Liu, B. (2010). Construction of membership functions for predictive soil mapping under fuzzy logic. Geoderma, 155(3-4), 164-174. 